\title{
Deux concepts de compromis : commun vs analogique
}

\author{
Mohamed Nachi ${ }^{1}$ \\ Institut des Sciences Humaines et Sociales, \\ Université de Liège
}

Ne doit-on admettre l'idée de compromis que lorsqu'il existe des partenaires et que ces derniers procèdent à des négociations et acceptent de faire de concessions mutuelles pour aboutir à une forme d'accord ? Est-il inapproprié d'utiliser le terme de compromis pour parler d'entités abstraites ou de catégories de pensées opposées ? Cet article pose la question de savoir quel est le lien à établir entre deux acceptions du terme compromis, dans son sens commun et son sens analogique. Son ambition est de construire deux concepts opératoires visant à rendre intelligibles les réalités multiples du compromis.

Pour ce faire, il m'a semblé pertinent de partir du champ lexical du mot compromis pour envisager cette construction. Les concepts commun et analogique seront donc appréhendés dans leurs relations avec le sens commun et avec les usages variés selon les contextes. Chaque concept est ensuite abordé dans la perspective d'en délimiter le champ lexical et d'en déterminer les principales caractéristiques spécifiques.

\section{CHAMP LEXICAL}

II n'est pas de tâche plus délicate que d'essayer de définir et de circonscrire de façon précise et, de surcroît, d'essayer d'élever au rang de concepts scientifiques des termes courants, banals, comme ceux d'accord, entente, compromis, négociation, concession, arrangement, adaptation, etc., qui sont nés de besoins pratiques, contingents et, de ce fait, chargés de connotations sociales, historiques et politiques qui les rendent aussi ambigus qu'insaisissables. II est aussi difficile de définir les mots dont on se sert couramment et spontanément. Le langage ordinaire est en effet aussi fluide que polysémique.

Nous sommes si accoutumés à nouer des accords et à fabriquer des compromis que nous n'éprouvons pas le besoin d'y réfléchir, car, comme le dit Montaigne, "l'habitude en ôte l'étrangeté ». Tout un chacun peut s'en servir sans nécessai-

1.M.Nachi@ulg.ac.be 
rement savoir ce qu'ils signifient plus précisément. Wittgenstein a raison de dire : « Comme il m'est difficile de voir ce que j'ai sous les yeux! » (Wittgenstein, 1990, p. 55). II est en effet difficile de se rendre compte de l'évidence, de ce qui s'étale sous nos yeux. De telles affirmations s'appliquent tout particulièrement au compromis. Celui-ci est, telle l'évidence de la coutume, partie intégrante de notre univers social, de notre monde commun. Prendre la mesure de son importance et, autant que faire se peut, le rendre intelligible, paraît être une tache semée d'embûches.

L'analyse des différents termes que couvre le champ lexical du terme compromis montre qu'il existe un ensemble d'occurrences qui entretiennent des relations réciproques. Il s'agit d'un réseau de significations à la fois lié et différencié, dont l'étude permet d'éclairer le sens de chaque vocable, mais aussi le sens de leurs relations dans le même champ lexical.

Ainsi, entre les termes déjà cités (arrangement, entente, etc.), il existe un ensemble d'occurrences dont les significations gravitent autour de l'idée commune d'acceptation, de transaction, d'accommodement entre ceux qui se mettent d'accord. Pour aboutir à la conclusion d'un accord, parvenir à un compromis, il faut préalablement passer par des discussions, négociations, échange de vues, transactions, etc., qui sont le prélude à la formation de cette forme d'accord ; à une entente, alliance ou rapprochement. Le sens générique du mot accord suppose l'établissement de quelque chose de commun, c'est-à-dire l'association, la convergence, la compatibilité entre des éléments différents. Dire que des partenaires se sont mis d'accord, c'est dire qu'ils sont arrivés à s'entendre, à avoir un avis commun, une intention ou opinion convergente ; à agir en commun. C'est, par exemple, le cas lorsqu'on parle d'accord à l'amiable, ou de gré à gré ; ou lorsqu'il est question d'accord conclu entre partenaires sociaux prenant la forme d'une convention collective, d'un compromis sur les salaires ou le temps du travail.

C'est, à mon sens, en partant de l'analyse de ce réseau de significations qu'il devient possible d'opérer un passage du terme commun de compromis, dont l'usage est répandu dans le langage ordinaire, à un véritable concept. Ce passage suppose une opération d'abstraction (raisonnement/procédure) ayant pour fonction de mettre en évidence ses aspects communs les plus saillants. Comment procéder pour l'élever jusqu'au niveau conceptuel - donc scientifique?

\section{DU MOT AU CONCEPT}

Le travail de conceptualisation est indispensable à toute entreprise scientifique qui prétend stimuler notre faculté d'étonnement et aller au-delà de nos préjugés et habitudes de penser. C'est que l'activité scientifique « s'accompagne d'une très forte dépendance théorique » (Schlanger, 1991, p. 68). L'élaboration conceptuelle obéit donc à un raisonnement et à des procédures qui sont propres à ce type de travail intellectuel. Comme l'affirment Gilles Deleuze et Félix Guattari : 
« Les concepts ne nous attendent pas tout faits, comme des corps célestes. II n'y a pas de ciel pour les concepts. Ils doivent être inventés, fabriqués ou plutôt créés, et ne seraient rien sans la signature de ceux qui les créent » (Deleuze et Guattari, 1991, p. 11).

Ainsi donc, le concept de compromis est d'une certaine manière à inventer, pour lui conférer le statut d'opérateur de représentations théoriques. Comment procéder? En partant du champ lexical initial, des usages du langage ordinaire, mais en reconstituant le champ sémantique du mot compromis, en l'inscrivant dans une perspective pragmatique capable d'en faire une « idée neuve et féconde », seIon l'expression de Claude Bernard. L'approche pragmatique s'intéresse plus particulièrement à la mise à l'épreuve du langage, de l'action et des justifications des acteurs ordinaires dans des situations de la vie quotidienne (Nachi, $2001 ; 2006)$. En l'occurrence, elle se préoccupe de la manière dont le compromis se déploie dans la pratique, tel qu'il est mis à l'épreuve, en actes, par les acteurs ; qui en construisent des figures multiples, en fonction des épreuves et situations sociales, leur conférant les justifications et légitimités qui conviennent (Boltanski et Thévenot, 1991 ; Thévenot, 1996).

En s'appuyant sur cette approche, l'enjeu est de parvenir à la fabrication d'un concept opératoire qui permet de rendre intelligible la réalité qu'il décrit. Toutefois, comme le souligne Geneviève Médevielle :

« Une notion ne saurait être traitée comme un simple contenant portant l'étiquette de son contenu et qui serait indifférent aux usages qu'on en fait. Or, la notion commune de compromis n'est pas univoque selon les pratiques qu'elle décrit, ni selon les utilisateurs, qui la chargent péjorativement ou qui lui attribuent un sens positif. La notion commune de compromis implique que quelque chose se passe entre deux partenaires pour que soit trouvé un accommodement entre des intérêts différents, voire opposés. " (Médevielle, 1998, p. 205)

Il importe, pour s'en tenir à la perspective pragmatique, de prendre en considération les usages différenciés du terme en fonction des contextes et de ses significations en actes, c'est-à-dire de sa performance, de son emploi effectif dans des situations concrètes. Cela passe par l'explicitation des relations triangulaires réciproques qui existent entre le terme commun de compromis, ses différents usages et sa conceptualisation potentielle. Cette relation triangulaire peut être représentée schématiquement comme suit :

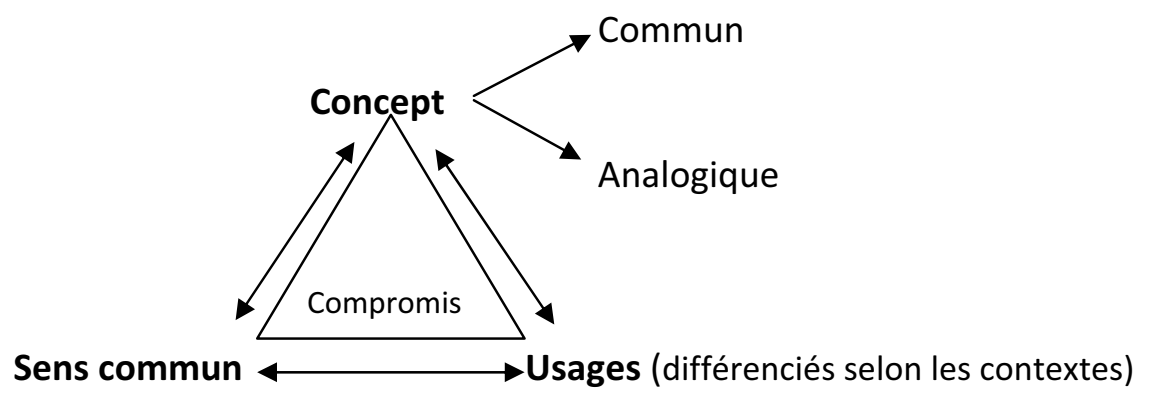


Bien évidemment, les usages du terme compromis sont multiples et variés et il serait vain de tenter d'en faire une énumération exhaustive. II est possible, en simplifiant, de distinguer les principaux domaines d'usage - juridique, économique, social et politique - au sein desquels le compromis peut revêtir des acceptions spécifiques. Entre ces différents usages, il est possible d'identifier un champ lexical commun, sorte de noyau de significations présent quel que soit l'usage et quel que soit le domaine d'application. Ce qui est commun, c'est un processus qui s'instaure entre des partenaires cherchant à parvenir à un accord au prix de quelques accommodements, adaptations et concessions réciproques entre des intérêts divergents. Je propose de désigner ainsi ce premier type de compromis : le concept commun de compromis.

Cependant, tout un pan des usages possibles reste non couvert par ce premier type de concept. II suffirait de penser à tous les usages renvoyant à des constructions intellectuelles et des configurations sociales et historiques qui, tout en étant constitutives de formes de compromis, ne supposent ni accords formels entre partenaires, ni négociations ni concessions réciproques. Pour couvrir ces multiples domaines d'utilisation, je propose de recourir à un usage analogique et métaphorique du concept commun. II s'agit d'un second type de concept, que je nomme : le concept analogique de compromis. II faut maintenant revenir sur ces deux types de concept, pour apporter davantage de précisions et en élucider les représentations théoriques.

\section{CONCEPT COMMUN}

L'inventaire des différentes occurrences du mot compromis permet d'identifier un champ sémantique commun aux divers contextes dans lesquels il est utilisé. Dans la pratique, le compromis implique une forme d'échange, d'interaction entre des partenaires mus par l'idée de parvenir à un accord, de trouver un accommodement entre des principes ou des intérêts contradictoires. Cela suppose, de la part des partenaires, une volonté de s'entendre, de coopérer ; un effort d'adaptation, de renoncement, de concessions réciproques, c'est-à-dire une volonté de se mettre d'accord pour composer et suspendre, ne serait-ce que momentanément, le différend ou la discorde. Dans cette perspective, le concept de compromis fonctionne comme une catégorie sociologique que l'on peut mobiliser pour appréhender des réalités multiples : sociales, politiques, économiques, culturelles, axiologiques, etc.

Ainsi, par exemple, dans la sphère politique, les discussions et délibérations dans l'espace public peuvent faire l'objet de compromis. De même, les tractations et alliances entre factions ou partis politiques peuvent déboucher sur des accords que l'on qualifie de compromis politique (Carens, 2011).

Dans la sphère économique et sociale, dans le monde du travail ou au sein de l'entreprise, la réponse à des revendications ou le règlement de certaines relations conflictuelles aussi bien individuelles - par exemple entre un contremaître et un ouvrier - que collectives - entre partenaires sociaux : syndicats et patronat - nécessitent le 
recours à des négociations pour parvenir à un accord ou une convention collective dont l'aboutissement passe par des transactions et des concessions mutuelles ayant pour prétention la formation d'un compromis satisfaisant (Nachi et de Nanteuil, 2006, voir la $2^{\mathrm{e}}$ partie «Compromis et régulations »).

Dans la vie quotidienne, plus généralement, les relations sociales étant faites, entre autres, de frictions, tensions, disputes, divergences et parfois même de conflits ouverts, il faut que des compromis entre positions antagonistes soient construits. Les relations en sein de la famille, entre voisins ou même entre des individus qui ne se connaissent pas, mais qui se trouvent dans une situation d'interaction, font parfois l'objet de discordes ou disputes dont le règlement pacifique passe par l'élaboration de compromis. II apparaît clairement que conflit et compromis vont de pair ; ils sont, comme le dit Simmel, dans « un rapport formel », et l'un (le compromis) permet d'achever l'autre (le conflit) (Simmel, 1990, p. 340).

Le compromis en tant que concept commun peut donc être considéré comme un mode de résolution de conflit ou, plus généralement, comme une forme de régulation sociale, c'est-à-dire un moyen de maintien de la paix civique entre des partenaires en situation de désaccord ou de conflit. Il en est ainsi des compromis noués dans le monde domestique, entre groupes sociaux ou entre organisations politiques, etc. La répartition des taches ménagères entre les membres de la famille est l'objet de compromis pour éviter les disputes et permettre à chacun de prendre part dans l'organisation et la réalisation des activités domestiques. Entre États, la coopération et le règlement de certains conflits passent aussi par l'élaboration de compromis.

Dans cette acception, le compromis est une pratique courante, ordinaire. En songeant aux travaux de Michel de Certeau, il serait sans doute intéressant de voir dans quelle mesure le compromis peut s'apparenter à une « invention du quotidien », un " art de faire », finalement assez proche des traits dominants de la culture ordinaire dont de Certeau a réussi avec brio à dépeindre les «logiques opératoires » 2 . Ces logiques opératoires ne sont pas sans rappeler celles qui animent le compromis en tant que pratique d'ajustement des actions ou décisions aux situations et, de fait, constitue un effort incessant de régler au plus juste des désaccords, des disputes ou des conflits. L'intérêt de ce rapprochement entre ces « arts de faire » propres au monde ordinaire et le sens commun du compromis est de comprendre à la fois l'importance de la place du compromis dans la vie quotidienne et la logique qui est au fondement des pratiques et activités sociales.

II apparaît ainsi clairement que l'existence du compromis est liée à l'existence des relations humaines. C'est à ce titre que Georg Simmel a pu parler de « l'une des plus grandes inventions de l'humanité » (Simmel, 1999, p. 341). De toute évidence, le compromis est consubstantiel au monde social et, de surcroit, inextricablement mêlé aux activités humaines les plus élémentaires. II sommeille, si l'on peut dire, dans les replis des relations sociales. II fait partie des pratiques, des techniques

2. Rappelons que Michel de Certeau (1990) reprend trois traits dominants qui sont des manières d'inventer le quotidien : la tactique, la «perruque », le braconnage. 
que les humains utilisent tout naturellement pour ajuster leurs actions, former des accords, prévenir des disputes ou régler des différends.

De ces considérations, on peut extraire les oppositions entre ce qui est associé et ce qui est opposé à l'idée de compromis :

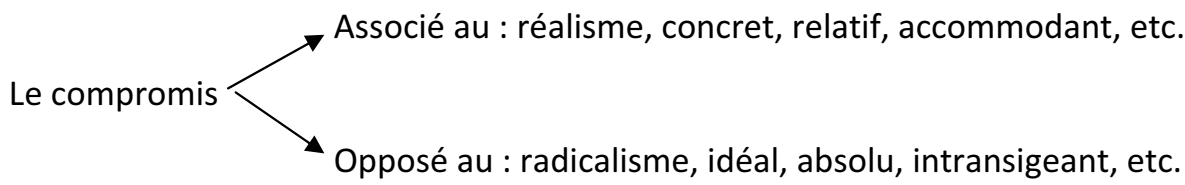

Cependant, les pratiques du compromis peuvent occuper une place plus ou moins grande en fonction de l'organisation sociale et politique et des traditions culturelles. Ainsi, par exemple, dans le contexte de la société française marquée par le jacobinisme, l'omniprésence d'un État central fort et d'une organisation administrative très hiérarchisée où la prise de décision provient des sommets de l'État (démarche de type «top - down »), la place réservée au compromis se trouve fortement réduite. En revanche, cette place est nettement plus importante dans des pays comme la Belgique, l'Allemagne, et plus encore dans les pays anglosaxons marqués par le pragmatisme. II s'ensuit, comme le fait remarquer Ernst Troeltsch lors d'une conférence prononcée en Grande Bretagne à la fin de sa vie (Troeltsch, 1990), que l'usage de ce terme est moins péjoratif en anglais qu'en allemand. Et d'ajouter : " II est plus facile pour moi de confesser ici mon adhésion au principe de compromis que dans mon propre pays » (Troeltsch, cité dans Médevielle, 1998, p. 207). C'est que la Grande Bretagne, «marquée par un certain pragmatisme politique et par l'empirisme philosophique, ne déprécie pas le compromise » (Médevielle, 1998, p. 207). Ce n'est peut-être pas par hasard si l'auteur qui a écrit le premier traité sur le compromis fut un Britannique : Lord John Morley qui publia, en 1874 à Londres, On Compromise...

De même, dans la tradition républicaine américaine, le compromis bénéficie d'une place de premier plan en raison du rôle qu'il a pu jouer dans l'édification du système politique fédéral du pays marqué, justement, par le pragmatisme de ses fondateurs (Rakove, 1987). À cet égard, Francis Biddle écrit :

« II est bien sûr évident pour tout Américain que toute notre vie nationale est construite sur le compromis et que la grande institution, la Constitution américaine, de laquelle nous tirons la force de notre vie publique, est en elle-même une série de compromis, petits et grands : les compromis entres les États et le gouvernement fédéral, entre le pouvoir central et celui des périphéries ; entre, pour être réaliste, les intérêts sudistes des planteurs du Sud et ceux des fermiers et des pêcheurs de la Nouvelle Angleterre » (Biddle, 1957, p. 1-2).

Le compromis renvoie à des pratiques différenciées, à des réalités multiples ; il est l'art du possible. Pour des raisons de différences culturelles, historiques ou encore pour des raisons liées au passé politique de chaque pays, il apparaît donc 
indispensable de faire la distinction entre les divers usages du compromis et les façons plus ou moins dépréciées de sa place dans l'espace social et politique. Le compromis est une forme sociale dont la cristallisation et la stabilisation s'ajustent au sens des réalités.

J'ai proposé de parler dans ce cas de concept commun dans la mesure où le compromis se révèle être une marque des relations humaines, une activité pratique indispensable au maintien du lien social, au déroulement des échanges et transactions de toutes sortes. II suppose des actions réciproques, des transactions, négociations, coopérations, renonciations, concessions, etc.

Cependant, ce concept n'est valide que pour parler de certaines situations à l'exclusion d'autres. Il est, en effet, des situations où le compromis ne suppose ni échanges immédiats entre des partenaires, ni concessions explicites ou négociations. Par conséquent, le concept commun dont on vient de préciser les contours, s'avère inapproprié parce que le compromis renvoie alors moins à des pratiques existantes qu'à une configuration idéelle. Pour rendre compte de celle-ci, je propose de recourir à un usage métaphorique et analogique du terme compromis.

\section{CONCEPT ANALOGIQUE}

Aux usages communs du compromis, il convient donc d'ajouter les utilisations analogiques qui ne se réfèrent pas nécessairement à des échanges ou à des activités sociales, mais renvoient à des processus de constructions intellectuelles et symboliques, à des représentations événementielles qui combinent, agencent et articulent des idées, des principes, des visions du monde, des valeurs, des pratiques, distincts. De la rencontre ou de la confrontation de ces éléments hétérogènes émerge un procès dialectique qui, de la dualité de deux termes antinomiques, fait naître un nouvel espace du possible, sorte de synthèse hybride dont la particularité est de dépasser la somme des éléments initialement en présence. C'est le cas, par exemple, de l'opposition entre tradition et modernité, entre l'idéal et le réel, entre l'universel et le particulier, etc. Le compromis s'avère ici une configuration intellectuelle qui, à un moment donné, se cristallise et se stabilise, donnant lieu à la formation d'une forme sociale sui generis. Tension, contradiction, relation dialectique, syncrétisme, adaptation, accommodation créative, etc., sont les termes par lesquels se caractérise ce type de compromis.

Le compromis exprime des tensions entre $\left\{\begin{array}{l}\text { Littéral / métaphorique } \\ \text { Idéal / réel } \\ \text { Universel / particulier } \\ \text { Absolu / relatif } \\ \text { Moderne / traditionnel }\end{array}\right.$


Ce genre de compromis n'implique ni négociations ou transactions formelles, ni concessions, au sens propre du terme, et encore moins un quelconque accord en bonne et due forme.

"Avec de tels compromis, ce n'est plus tant la notion d'accord et de coopération qui domine, mais celle de Spannung. La "tension" devient un élément caractéristique pour penser le compromis. Avec une telle utilisation du mot "compromis" dans un sens analogique, pour parler de la relation entre deux pôles antonymiques, une dualité commence à apparaître : le compromis est d'une part l'expression d'un vécu et d'une pratique, mais d'autre part il exprime aussi une conception déjà objectivée de relations, non sans lien analogique avec la pratique, mais susceptible de la déborder largement. Un nouvel espace de pensée est ouvert par le procès analogique » (Médevielle, 1998, p. 209).

La dimension analogique du compromis appelle quelques commentaires et précisions afin d'en mesurer la portée. Pourquoi en effet recourt-on à l'analogie? L'enjeu est épistémologique dans la mesure où la prétention est de construire un concept opératoire susceptible de rendre compte d'une configuration intellectuelle composite qui, sans ce procès analogique, serait restée insaisissable d'un point de vue théorique. Le raisonnement analogique permet, on le sait, d'établir par l'imagination des correspondances, des rapprochements fondés sur des rapports de similitudes entre plusieurs objets de pensée différents. La linguistique fournit une illustration de ce procès analogique : c'est l'action assimilatrice qui fait que certaines formes (langagière, sémantiques, etc.) changent sous l'influence d'autres formes auxquelles elles sont associées dans l'esprit et qui détermine des créations conformes à des modèles préexistants. À cet égard, l'analogique se fonde sur des rapports de correspondance et non sur un principe d'identité, ce qui fait son caractère imparfait. II relève de ce que Judith Schlanger appelle la « pensée inventive ». Selon cet auteur :

«La réussite de l'analogie ne consiste pas à développer systématiquement, trait à trait, le détail des correspondances. Et une correspondance imparfaite n'est pas nécessairement l'échec de l'analogie. En disant que le modèle analogique est moins une représentation qu'un langage, on déplace sa réussite et son échec du côté de ce qu'il rend possible, du côté de ce qu'il donne à dire. La fonction des modèles analogiques n'est pas directement cognitive mais avant tout heuristique : elle est de donner à voir et de donner à dire, d'étendre et d'enrichir l'espace traitable » (Schlanger, 1991, p. 86).

Le concept analogique de compromis est donc un concept heuristique qui, en partant d'une correspondance imparfaite, permet d'étendre l'analyse à des configurations intellectuelles ou historiques constituées d'éléments contradictoires, voire opposés et traversés par des tensions : entre absolu / histoire, universel / particulier, idéel / matériel, passé / présent, tradition / modernité, etc. Ainsi, en se basant sur le raisonnement analogique, il devient possible d'effectuer

« un rapprochement et un rapport de convenance entre la notion commune de compromis, qui suppose une médiation entre des intérêts opposés, et un type de relation entre pôles antinomiques. La convenance est sélective dans la me- 
sure où il n'y a pas correspondance exacte entre les deux types de compromis 》 (Médevielle, 1998, p. 209).

Une illustration significative peut être trouvée dans la manière dont Ernst Troeltsch se sert de la notion de compromis pour penser la relation du christianisme au monde et à la culture, la relation de l'Absolu à l'histoire ; la relation entre religion et société. À ses yeux, le concept de compromis permet justement d'instituer l'Absolu dans l'histoire ; il est ce qui caractérise le cours de l'histoire ${ }^{3}$. Prenons un autre exemple tiré du contexte islamique. À ce propos, il me semble approprié de se servir de ce concept analogique pour parler du courant réformiste qui s'est développé au XIX ${ }^{e}$ siècle dans les sociétés musulmanes. On sait que le réformisme est un mouvement intellectuel qui a émergé suite aux contacts ou la confrontation du monde musulman avec l'Occident. Les penseurs musulmans, attirés par les idées modernes (liberté, égalité) et par le progrès technique entendaient montrer la nécessité pour les sociétés musulmanes de se moderniser, mais sans trahir leur histoire ou oublier leur héritage (turâth). II n'est donc pas surprenant que ces auteurs aient cherché à concilier certaines idées, valeurs et institutions inspirées des sociétés occidentales avec celles propres aux sociétés musulmanes. Ils déployèrent de grands efforts pour introduire des changements institutionnels, politiques, culturels, intellectuels, etc., en prônant des solutions de compromis. Ainsi, ils s'efforcèrent de penser un nouveau système juridique qui, tout en respectant les principes de la Shari'a (Loi musulmane), confère une large place au droit positif d'inspiration européenne. Dès lors, de manière générale, ce réformisme peut être considéré comme une configuration idéelle, un compromis intellectuel, puisant ses sources tout autant dans la pensée et la tradition islamiques que dans la modernité occidentale. II s'agit d'un compromis élaboré par les réformistes musulmans pour rendre compatible les relations entre turâth (tradition) et hadâtha (modernité) ; relations parfois conflictuelles, mais travaillées et marquées par l'interpénétration d'idées et des valeurs issues de deux visions différentes. L'usage du terme compromis est donc métaphorique dans la mesure où il ne s'agit pas d'un accord formel et son élaboration n'est pas le fruit d'une négociation entre partenaires. Dans ce compromis, il n'est pas question de « couper la poire en deux », comme on dit souvent, parce que le compromis ne se résume pas à une question de justice distributive. II est l'expression d'un rapprochement d'idées créant un nouvel espace de pensée ouvert à des pratiques, principes et valeurs hétérogènes.

Un tel compromis est parfois dénoncé, au nom d'une certaine authenticité de la tradition ou des valeurs «pures » de l'islam, comme une « compromission des idées ». Mais les caractéristiques des sociétés musulmanes contemporaines, leurs traits culturels actuels montrent que le « compromis réformiste » est devenu une configuration historique dans la mesure où il exprime un mode d'être de ces sociétés ; tout en demeurant musulmanes, ces sociétés sont désormais devenues occidentalisées, à des degrés divers.

3. Cette thématique est récurrente dans l'œuvre de Troeltsch (Troeltsch, 1996, p. 10-19; 1990, p. 229-262). 


\section{EN GUISE D'OUVERTURE}

Au total, il apparaît que les deux concepts de compromis ont des ressemblances qui se chevauchent, mais ils ne se confondent pas ; ils ont ce que Wittgenstein appelle un « air de famille ». Cette notion est extrêmement importante pour préciser notre façon de considérer les concepts que nous utilisons. D'une part, elle permet d'éviter de succomber à la tentation, qui guette tout chercheur, d'essentialiser les concepts dont il se sert. D'autre part, elle suppose de reconnaître le caractère « vague » de bon nombre de termes que nous utilisons et qui ne peuvent pas avoir de définition stricte. Enfin, la notion d'air de famille implique une pensée inventive permettant de faire des rapprochements entre les usages différenciés d'un même concept. Wittgenstein soutient justement « qu'il n'est pas nécessaire, pour comprendre ou expliquer un concept, de pouvoir en donner une définition caractère par caractère, ce qui impliquerait que le concept en question ait des bords nets. Le plus souvent nous utilisons les termes conceptuels sans signification nettement délimitée ; c'est notamment le cas du concept de jeu qui est le concept d'air de famille par excellence. Un réseau compliqué de ressemblances qui se chevauchent et s'entrecroisent unit les jeux et nous autorise à parler du concept de jeu » (cité dans Chauviré et Sackur, 2003, p. 5).

La réflexion sur le compromis se doit de s'éloigner des logiques d'opposition et des positions antinomiques, car le propre de l'idée de compromis est d'éviter les extrêmes et toutes les formes de radicalité. Elle suppose que l'on s'écarte de toutes les espèces d'essentialisme afin de chercher ses fondements dans une « raison pragmatique ». La renonciation à la « solution parfaite » est dès lors ce qui caractérise la recherche de compromis. Celui-ci est toujours un moyen terme, une construction hybride dont les manifestations relèvent de plusieurs ordres de réalité, d'une pluralité des ordres de grandeur, selon les termes de Luc Boltanski et Laurent Thévenot (1991). Dès lors, l'usage du concept de compromis, au sens à la fois commun et analogique, ne peut prétendre couvrir une quelconque forme de radicalité dans la mesure où il exclut les positions tranchées, les attitudes intransigeantes ou absolutistes. En outre, le compromis idéel, en tant que configuration historique - à l'instar du « compromis réformiste » cité plus haut -, ne peut être une " solution parfaite », mais la réponse contingente à la tension qui existe entre des éléments hétérogènes appartenant à des univers symboliques et des répertoires socioculturels et politiques différents. II est une forme de médiation opérant un rapprochement entre pôles antinomiques.

Le compromis est le produit de processus contradictoires continus, mais inachevés, dont l'aboutissement n'est jamais prédéterminé à l'avance. Pour cette raison, la part du hasard dans la construction sociale du compromis est plus grande que celle de la nécessité. La place du hasard est aussi grande dans la mesure où, une fois engagé, l'issue de tout processus de formation de compromis demeure incertaine ; on ne saurait savoir à l'avance à quel genre de compromis nous aurons affaire ! Cette indétermination est ce qui fait son originalité, mais aussi sa fragilité et son caractère inachevé. L'idée de processus perpétuel et de dynamique incessante à l'œuvre dans la construction de compromis supposent sa réversibilité et font de lui une configuration en perpétuelle évolution, jamais définitive, jamais close. 
D'un point de vue axiologique, le compromis incarne un pluralisme des valeurs, ce qui exclut toute vision moniste. À cet égard, l'une de ses caractéristiques est de s'enraciner dans une anthropologie réaliste de l'agir humain et des valeurs socioculturelles et politiques. Comme le souligne Robert Grimm :

"Le compromis s'inscrit comme un principe de réalité. II se soucie des médiations, de la praticabilité, des conséquences. II prend acte des conditionnements et des limites. II s'impose par la nécessité de gérer des alternatives, des oppositions, des écarts. Celui qui y consent ne veut ignorer ni la complexité des situations, ni la pluralité des réponses, ni la relativité des connaissances et des normes »(Grimm, 1984, p. 260).

Par ailleurs, le compromis enseigne qu'il ne peut pas y avoir de morale absolue et que, pour des raisons inhérentes à la condition humaine, les choix moraux, les décisions et actions fondées sur le compromis ne sont jamais irréversibles; bien au contraire, ils s'accompagnent, la plupart du temps, d'un sentiment d'imperfection et d'incomplétude. De là découlent l'ambivalence du compromis et son caractère équivoque. D'une certaine manière, le compromis révèle le côté ambivalent et tragique de l'action humaine.

Cependant, cette ambiguïté n'affecte en rien le caractère dynamique du compromis, dans la mesure où il doit demeurer en perpétuelle réévaluation; il ne peut se figer en une prétendue action, norme ou décision définitive, puisqu'il s'agit par définition d'un processus inachevé. C'est du reste pour cette raison que le compromis se situe à contre-pied de toute logique du « ou bien, ou bien », d'une morale du « tout ou rien », ou encore du « tout pour le tout ». ${ }^{4}$ Le compromis apparaît non seulement comme une catégorie éthique qui place au premier plan les sentiments d'incomplétude et d'humilité, mais encore comme une " promesse » (Nachi, 2003) qui, comme toute espérance, préfigure la volonté de s'entendre et de vivre avecles autres.

\section{RÉFÉRENCES}

BIDDLE Francis (1957), « Necessity of Compromise », dans Robert M. Mac Iver (ed.), Integrity and Compromise. Problems of Public Private Conscience, New York, Harper \& Brothers.

BOLTANSKI Luc et Laurent THÉVENOT (1991), De la justification. Les économies de la grandeur, Paris, Gallimard.

CARENS Joseph (2011), "Le compromis en politique », dans Mohamed Nachi (dir.), Actualité du compromis. La construction politique de la différence, Paris, A. Colin, p. 16-36.

ChauviRÉ Christan et Jean SACKUR (2003), Le vocabulaire de Wittgenstein, Paris, Ellipses.

4. Au sujet de cette « loi du tout-ou-rien » en tant que morale intransigeante, on se reportera avec intérêt à l'analyse que lui consacre Vladimir Jankélévitch (1981, p. 52). 
Certeau (DE) Michel (1990), L'invention du quotidien. 1. Arts de faire, Paris, Gallimard. Deleuze Gilles et GuATTARI Félix (1990), Qu'est-ce que la philosophie ?, Paris, Minuit. GrIMm Robert (1984), L'institution du mariage. Essai d'éthique fondamentale, Paris, Cerf.

JANKÉLÉVITCH Vladimir (1981), Le paradoxe de la morale, Paris, Seuil.

MÉDEVIELLE Geneviève (1998), L'absolu au cœur de l'histoire. La notion de compromis chez Ernest Troeltsch, Paris, Cerf.

$\mathrm{NACHI}$ Mohamed (2001), "La vertu du compromis : Dimensions éthique et pragmatique de l'accord ", Revue interdisciplinaire d'études juridiques, $n^{\circ} 46$, p. 81-110.

$\mathrm{NACHI}$ Mohamed (2003), Éthique de la promesse. L'agir responsable, Paris, PUF.

$\mathrm{NACH}$ Mohamed (2006), Introduction à la sociologie pragmatique, Paris, A. Colin.

NACHI Mohamed (2011), Actualité du compromis. La construction politique de la différence, Paris, A. Colin.

NACHI Mohamed et Matthieu de NANTEUIL (dir.) (2006), Éloge du compromis. Pour une nouvelle pratique démocratique, Louvain-la-Neuve, Academia Bruylant.

RAKOVE Jack N. (1987), " The Great Compromise: Ideas, Interests, and the Politics of Constitution Making », The William and Mary Quarterly, vol. 44, n 3, p. 424-457.

SIMMEL Georg (1999), Sociologie. Essai sur les formes de la socialisation, Paris, PUF.

SCHLANGER Judith (1991), «La pensée inventive », dans Elisabeth Stengers et Judith Schlanger, Les concepts scientifiques. Invention et pouvoir, Paris, Gallimard.

THÉVENOT Laurent (1996), « Justification. Justification et compromis », dans Monique Canto-Sperber (dir.), Dictionnaire d'éthique et de philosophie morale, Paris, PUF, p. 789-794.

TroeltSCH Ernst (1990), Religion et Histoire, Genève, Labor et Fides.

TROELTSCH Ernst (1996), Histoire des religions et destin de la théologie, Paris et Genève, Cerf-Labor et Fides.

WitTGenstein Ludwig (1990), Remarques mêlées, Paris, Gallimard. 